\title{
La presencia de las cooperativas de Catalunya en Internet
}

\author{
Alex Araujo Batlle \\ Eloi Serrano Robles \\ Víctor Jordan Vallverdú
}

RESUMEN: Internet se ha convertido en la electricidad del siglo XXI. La presencia en la red es un imperativo para cualquier empresa que quiera ser encontrada. Además, factores como la consolidación del uso de dispositivos móviles como ventanas para acceder a la red y los hábitos de las generaciones que se incorporan al mercado, refuerzan la necesidad de considerar la presencia en la red para cualquier empresa, incluidas las cooperativas. El presente trabajo analiza la presencia web de 3860 cooperativas de Catalunya a través la combinación de técnicas cuantitativas y cualitativas. Los resultados apuntan que dos de cada tres cooperativas no disponen de una página web propia, siendo la falta de recursos, de formación y el poco soporte institucional las principales causas de estos datos. El presente trabajo pretende ser una herramienta para conocer el estado actual de las cooperativas en internet y una aproximación para enfocar las estrategias de futuro.

PALABRAS CLAVE: Internet web, generación digital, comunicación, información, cooperativas.

CLAVES ECONLIT: D83, L86, M31, 000, Q55.

Cómo citar este artículo / How to cite this article: ARAUJO, A., SERRANO, E. \& JORDAN, V. (2020): "La presencia de las cooperativas de Catalunya en Internet”, CIRIEC-España, Revista de Economía Pública, Social y Cooperativa, 99, 37-56. DOI: 107203/CIRIEC-E.99.16902.

Correspondencia: Alex Araujo Batlle, aaraujo@tecnocampus.cat,

ORCID: http://orcid.org/0000-0003-0776-7557

Eloi Serrano Robles, eserrano@tecnocampus.cat

ORCID: https://orcid.org/0000-0002-3821-2944

Víctor Jordan Vallverdú, vjordan@tecnocampus.cat

ORCID: http://orcid.org/0000-0002-2683-3129

Tecnocampus - Universitat Pompeu Fabra (UPF) 


\section{EXPANDED ABSTRACT}

\section{The web presence of cooperatives in Catalonia}

In 2019, $75 \%$ of Spanish SMEs had a website (ONTSI, 2020) and the number of domain names registered worldwide in the first quarter of 2019 alone was 351.8 million (Verisign, 2019). There is no doubt that the Internet has become the electricity of the 21 st century. An online presence is imperative for any business that wants to be found.

In this scenario, we cannot forget a factor that has changed the way we access the web: the mobile device. Since 2016 the leading search engine, Google, announced that it would penalize in the search results those web pages that were not optimized for mobile devices, adopting a mobile-first policy. Considering that Google has maintained a market share of close to $90 \%$ for a decade, it is essential that web pages must be adapted to mobile devices.

Finally, a third factor to take into account is the relevance of identity on the web (Juliá et al., 2004). In December 2001, the IANA (Internet Assigned Numbers Authority), reported the implementation of the .coop domain to identify those websites belonging to cooperatives. Thus, since 2002 this domain is reserved for those websites belonging to entities constituted as cooperatives.

There are currently 7,919 .coop domains registered worldwide, a very modest figure if we compare them with the 108,000 .cat domains, the nearly 1.9 million .es domains, 10 million .org domains, 13.5 million .net domains or the more than 142 million .com domains registered in 2019.

We have numerous studies carried out periodically on the use of technology and the presence of SMEs on the Internet. However, this monitoring, which allows us to obtain an X-ray of the evolution of the business environment, does not let us know the specific development of the cooperative sector. Do they follow similar patterns? Are the levels of presence on the Internet of the cooperatives similar to those of the rest of the companies?

The aim of this paper is to address these issues within the framework of cooperatives registered in Catalonia. Specifically, it is addressed in two phases through a combination of quantitative and qualitative methodologies.

Firstly, and taking as a universe the 3,860 cooperatives registered in 2017 in the General Registry of Cooperatives of Catalonia, a content analysis is carried out to complete the information on the cooperatives registered with a website. In cases where a corporate website is identified, the domain extension used is determined, taking into account the classifications defined by the IANA. To conclude this first phase of the analysis, and taking into account that the Mobile Checker tool developed by the 
World Wide Web Consortium, the consortium in charge of developing and guaranteeing compliance with the use of web standards, has been disabled for discontinuity since 2016, a test is carried out to adapt the website to mobile devices using Google's Search Console tool.

After this first phase of analysis, a second phase is planned based on a survey sent to all those cooperatives in which a website has been located. This survey aims to find out the reasons why these cooperatives have a presence on the Internet, to find out the level of satisfaction with the use of the network that they are making and to ask them if they think there is any difference, in relation to the presence on the Internet, because they are a cooperative. This survey was sent to 1,025 co-operatives that had a corporate operational website, obtaining a total of 136 responses.

The results of the first phase show that $27 \%$ of the total number of cooperatives registered in the General Register of Cooperatives of Catalonia have a corporate website. Bearing in mind that $89 \%$ of the sample of Catalan cooperatives have fewer than ten members, the reference figure would be the equivalent of microenterprises which, according to the FAEDPYME report (2018), have a web presence for $78.6 \%$ of them.

The next aspect analyzed was the adaptability of the website to mobile devices. After analyzing the 1,015 web pages of the cooperatives, we see that $52 \%$ of them are adapted to mobile devices according to Google's tool.

Finally, the online identity of the different cooperatives is analyzed. We found that the domain .coop is only being used by $6 \%$ of Catalan cooperatives with a website. Most of the cooperatives bet on the use of domains .com (46\%), .cat (20\%) and .es (14\%).

Secondly, and to complement the information collected through the analysis of content, the results of the surveys conducted on cooperatives with websites are analyzed. In this regard, the questions relating to the purpose, key elements and main barriers to having a website are addressed below.

Nine out of ten cooperatives reported using their website for three main reasons: a) to show what they do, the products and services; b) to have a presence on the net; $c$ ) to be reached.

Besides, three out of four cooperatives with a website say that this channel allows them to be closer to both customers and suppliers. On a scale of 0 to 5 , with five being very much in agreement and 0 being very much in disagreement, the cooperatives rank above three that having a website makes it easier for them to promote and sell products and services, to be closer to and improve B2B and B2C communication, and to improve strategy.

One of the perceptions that are repeated among the cooperatives surveyed is that there is considerable room for improvement. Furthermore, when asked if being a cooperative implies any difference in the use and presence in the network, the answer in $90 \%$ of the cases is no. 
So, if there is a clear difference between the penetration of the web presence in small and medium-sized enterprises that is much higher than in cooperatives, how can cooperatives be helped to narrow this gap? When asked about this question, cooperatives highlighted the lack of resources as the main reason. Training is, in turn, a major stumbling block for more than half of the respondents, while institutional support also represents a crucial aspect of being improved.

Resources are undoubtedly a vital issue in this equation, and it is particularly complicated if we take into account the size of most Catalan cooperatives: we are talking about a sample where most of the cooperatives are made up of three members.

This work offers a first approach to the web presence of cooperatives in Catalonia. The data obtained confirm the low maturity of the web presence of the cooperatives observed by Cristóbal et. Al. (2017) in oleic cooperatives.

There is ample room for improvement. This could be addressed from different perspectives. Firstly, and in response to demand from the cooperative members themselves, through training and information, for example, on success stories.

The principal value of this work is to establish a starting point. A framework that allows the intensification of studies on the presence in the network by the cooperative sector, helping its growth and competitiveness.

KEYWORDS: Internet Web; net generation; communication; information; cooperatives. 


\section{Introducción}

Seguramente internet representa uno de los proyectos cooperativos más importantes del siglo veinte. Cooperativo por el proceso de diseño de la red y por su estructura de funcionamiento descentralizada basada en protocolos abiertos de comunicación (Castells, 2001). La World Wide Web ha crecido de manera continuada especialmente desde la liberación del comercio a través de Internet en EUA a mediados de los noventa. Un ejemplo lo podemos encontrar en el número de nombres de dominio registrados: solo en el primer trimestre de 2019 se han registrado un total de 351,8 millones de dominios en todo el mundo (Verisign, 2019).

¿Hasta qué punto es importante la red en nuestra sociedad? El mismo Castells, en referencia a la importancia de esta, afirmaba: "Pero quizás exista otra opción. Me imagino que alguien podría decir: '¿Por qué no me deja usted en paz? Yo no quiero saber nada de su internet, de su civilización tecnológica, de su sociedad red'. Muy bien, pues si ese fuera su caso tengo malas noticias para usted: si usted no se relaciona con las redes las redes sí se relacionan con usted. Mientras quiera seguir viviendo en sociedad, en este tiempo y en este lugar, tendrá usted que tratar con la sociedad red. Porque vivimos en la Galaxia internet” (Castells, 2001: 292).

En este escenario no podemos olvidar un factor que ha cambiado la forma en como accedemos a la red: el dispositivo móvil. Son múltiples los indicadores que nos muestran como el dispositivo móvil se ha convertido en el dispositivo principal de acceso a Internet. Según Pew Internet Research, en enero de 2018 el $95 \%$ de los norteamericanos disponen de un teléfono móvil, siendo un $77 \%$ de ellos un Smartphone (Pew Research, 2018). A nivel global, la penetración del teléfono móvil supera el 95\%, y hasta en los países menos desarrollados (Least Developed Countries, LDC) la penetración de la telefonía móvil se sitúa en un 77\% (ITU, 2018). En España, el móvil es el dispositivo más usado para acceder a Internet, usado ya por el 94,6\% de los usuarios (Ditrendia, 2017).

Por si fuera poco, existe todavía otro factor clave para entender el escenario actual: la generación que irrumpe en el mercado. Desde la ya universal definición de Prensky (2001), numerosos autores han abordado la cuestión acerca de las características de las generaciones que han nacido normalizando la omnipresencia de internet en el día a día. Autores como Neil Howe o William Strauss afirman que se trata de una generación más numerosa, más agrupada, mejor educada y más étnicamente diversa (Howe \& Struass, 2000), mientras que otros autores hablan de perfiles completamente adaptados a la explosión comunicativa que ha significado Internet y la Web 2.0 (Lai \&y Hong, 2015; Lanier, 2011). Estas generaciones llevan el concepto de residentes y visitantes de White \& Le Cornu (2011) a su máxima expresión, integrando los artefactos tecnológicos como parte de su interfaz de interacción con el mundo (Palfrey \& Gasser, 2008; Tapscott, 1998). 
Vista la importancia de internet para las empresas, en 2019 el 75\% de las PYMES españolas tenían un sitio web (ONTSI, 2020), la familiarización con la red de las generaciones ya adultas, y el crecimiento de los dispositivos móviles como dispositivo de acceso, resulta relevante preguntarse hasta qué punto las cooperativas están preparadas para este escenario. Así, el objetivo de este trabajo es examinar la presencia en Internet de las cooperativas, concretamente el caso de Catalunya. Además, resulta interesante preguntarse si han apostado por esta presencia de forma consciente y estratégica, cuáles son los factores que han tenido en cuenta los miembros de las cooperativas para apostar por la presencia, o no, en internet, y si existe una percepción de singularidad en relación con este tema frente a otras empresas u organizaciones que no han estado constituidas como cooperativa.

Para afrontar estas cuestiones se ha realizado un estudio en dos fases. Para la primera fase se ha usado una metodología de análisis de contenido tomando como muestra las 3.680 cooperativas registradas en el Registro General de Cooperativas de Cataluña de 2017. En base a esta muestra, se ha analizado de cada una de ellas la presencia en internet, por sectores y por provincias, así como la adaptabilidad de sus páginas web a dispositivos móviles y al uso de extensiones de dominio específicas para las cooperativas. En una segunda fase, se ha realizado una encuesta a las cooperativas con presencia en internet preguntando por los motivos y las barreras que las han llevado a tener presencia en la red. En esta segunda fase se han mandado un total de 1.025 encuestas a todas las cooperativas con página web, y se han obtenido un total de 136 respuestas.

\section{Marco teórico}

Hace más de una década que se analiza la presencia en internet de las cooperativas en España. En 2004 Mozas y Bernal encuestaron a más del 37\% de las sociedades cooperativas de segundo grado españolas. En el citado documento, el $44,3 \%$ de las cooperativas respondieron que tenían una página web (Mozas \& Bernal, 2004). Ese mismo año, Juliá, García y Polo investigaron el tipo de información suministrada a través de la Red por parte de las cooperativas más representativas en diferentes sectores. Las conclusiones del trabajo apuntaban a la necesidad de aprovechar de una manera más efectiva las oportunidades que les ofrecía la red (Juliá et al., 2004). Un ejemplo de este margen de mejora lo encontramos en las cooperativas agrarias de la comunidad valenciana, en un estudio publicado solo dos años después, se mostraba que, en la provincia de Valencia, menos de un $10 \%$ disponía de una página web. Además, las cooperativas que habían implementado una web la usaban básicamente para describir su actividad empresarial (Juliá et. al., 2006).

Casos más recientes los encontramos en el análisis de las cooperativas oleícolas y su relación con las TIC. En 2015 un estudio mostraba como en este sector el uso de las TIC era bastante inferior 
en comparación con las empresas no cooperativas del sector (Fernández et al., 2015). En el caso concreto de las cooperativas oleícolas de Cataluña, se observaba un escaso grado de madurez en el desarrollo de los sitios webs (Cristóbal et al., 2017).

El Informe PYME España 2018 basado en una muestra de 1136 empresas, afirma que el 83,2\% de las PYME disponen de página web propia (FAEDPYME, 2018). Este dato disminuye hasta el $78,6 \%$ en el caso de las microempresas (menos de diez empleados). En este sentido, para poder comparar entre la madurez del sector de las cooperativas y el de las empresas con otras fórmulas jurídicas, resulta interesante tener en cuenta el número de socios de estas.

Otro aspecto relevante hoy en día, más allá de disponer de una página web, es el hecho de que esta esté adaptada para dispositivos móviles. Desde 2016 el buscador anunció que penalizaría en los resultados de las búsquedas aquellas páginas web que no estuvieran optimizadas para dispositivos móviles, adoptando una política mobile first ${ }^{1}$. Teniendo en cuenta que Google mantiene una cuota de mercado cercana al $90 \%$ desde hace una década ${ }^{2}$, resulta imprescindible que las páginas web estén adaptadas a dispositivos móviles.

Finalmente, no podemos olvidar la relevancia de la identidad en la red. Según Juliá et al. (2004) "un dominio como denominación de un nombre o dirección de Internet, identifica una empresa 0 un determinado producto en el ciberespacio, y facilita su localización, proporcionando información a millones de usuarios de la Red".

Tal como establece la ICANN (Internet Corporation for Assigned Names and Numbers) existen múltiples extensiones de dominio:

- Los Top Level Domain (TLD), de tres caracteres y que identifican una dirección con su actividad. Por ejemplo .com: comercial; .net: red; .org: para organizaciones; . edu: para instituciones educativas; .gov: para instituciones gubernamentales.

- Los Country Code Top Level Domain (cc-TLD), de dos caracteres que identifican el país en el que opera aquella página web. Por ejemplo .es: España; .uk: United Kingdom; .fr: Francia, it: Italia; etc.

- Los Geographic Top Level Domain (geo-TLD), sin restricción de caracteres que indican una zona geográfica que, no necesariamente se corresponda con un país oficial. Por ejemplo: .cat: Cataluña;.eus: Euskadi; .barcelona: Barcelona; etc.

- Los Generic Top Level Domain (g-TLD), dominios distintos de todos los anteriores, sin restricciones de caracteres y que pueden indicar la actividad o finalidad de la página web de una forma más concreta. Por ejemplo: .bike: para páginas relacionadas con bicicletas; . hotel: para hoteles; etc.

\footnotetext{
1.- Blog oficial de desarrolladores de Google <https://webmasters.googleblog.com/2016/11/mobile-first-indexing.html>

2.- Cuota de mercado de los principales buscadores <https://www.statista.com/statistics/216573/worldwide-market-share-of-search-engines/>
} 
En diciembre de 2001 la IANA (Internet Assigned Numbers Authority) informaba de la puesta en funcionamiento del dominio .coop para identificar aquellas páginas web pertenecientes a cooperativas $^{3}$. De esta forma, desde el 2002 este dominio está reservado para aquellas páginas web pertenecientes a entidades constituidas en forma de cooperativa.

Actualmente hay 7.919 dominios .coop registrados a nivel mundial, una cifra muy modesta si los comparamos con los 108 mil dominios .cat, los cerca de los 1,9 millones de dominios .es, 10 millones de dominios .org, 13,5 millones de dominios .net o los más de 142 millones de dominios .com registrados en $2019^{4}$.

Así, el objetivo principal de este trabajo es analizar la presencia en internet de las cooperativas, concretamente en el caso de las cooperativas registradas en Catalunya. Además de observar cuantas cooperativas tienen página web, según sectores y provincias, nos fijaremos también en la adaptabilidad de estas webs a los dispositivos móviles y a la identidad usada en internet. Finalmente, nos interesa explicar el motivo de estos resultados: cuales son las razones por las que las cooperativas están en internet y cuales son las principales barreras.

\section{Metodología}

El presente análisis se centra en la presencia de las cooperativas catalanas en Internet. Metodológicamente se ha optado por una triangulación metodológica que nos permite abordar la cuestión desde más de una perspectiva (Decrop, 1999).

La localización de los datos de las cooperativas se aborda a partir del Registro General de Cooperativas de Catalunya y tomando como universo las 3.860 cooperativas inscritas en 2017. En una primera fase se ha analizado la presencia en internet mediante una página web corporativa, su adaptabilidad a dispositivos móviles y el dominio usado. Para esta primera fase del análisis se ha usado una metodología de recogida de datos basada en el análisis de contenidos. El análisis de contenidos es una metodología descriptiva basada en la observación de las pautas más comunes en estudios de contenidos (Palacios \& Díaz Noci, 2009), en este sentido permite una descripción objetiva, sistemática y cuantitativa del contenido de las comunicaciones (Berelson, 1952) siendo, en la actualidad, una metodología empleada para el análisis de comunicaciones en múltiples formatos (López-Noguero, 2002).

3.- Comunicado de la IANA sobre dominios .coop < https://www.iana.org/reports/2001/coop-report-13dec01.html >

4.- Consulta de dominios registrados según su extensión < http://research.domaintools.com/statistics/tld-counts/> 
Uno de los datos recogidos a través del análisis de contenido es la adaptabilidad de las páginas webs a dispositivos móviles. Puesto que la herramienta Mobile Checker desarrollada por el World Wide Web Consortium, el consorcio encargado de desarrollar y garantizar el cumplimiento del uso de estándares web, se encuentra deshabilitado por discontinuidad desde 2016 (https://www. w3.org/2016/11/mobile-checker-disabled/), el análisis se ha realizado a través de la herramienta Search Console de Google.

La muestra agrupa las 3.680 cooperativas, el 100\% del universo del Registro General de Cooperativas de Catalunya, en cuatro sectores: agricultura, construcción, industria y servicios. El $60 \%$ de las cooperativas de la base de datos forman parte del sector servicios siendo un total de 2.320 . El sector de la construcción agrupa al 17\% de cooperativas (644), el de la industria el 16\% (611) y el sector agrícola el $7 \%$ (285). Por provincias, Barcelona agrupa al $72 \%$ del total de cooperativas (2788), Tarragona (430) y Lleida (416) representan un $11 \%$ cada una, y Girona agrupa al $11 \%$ de cooperativas (226).

\section{Tabla 1. Análisis descriptivo de la muestra}

\begin{tabular}{|l|l|}
\hline Número de socios & Indicador \\
\hline 16,21 & Promedio \\
\hline 141,85 & Desviación estándar \\
\hline 5.395 & Máximo \\
\hline 1 & Mínimo \\
\hline 3 & Moda \\
\hline 3 & Mediana \\
\hline
\end{tabular}

FUENTE: Elaboración propia.

Con el objetivo de explicar los resultados de la fase anterior y recoger la visión cualitativa de las cooperativas, en una segunda fase del análisis se ha usado una metodología cualitativa basada en encuestas. A partir de los resultados del análisis de contenidos se han distribuido formularios por medios electrónicos a las 1.025 cooperativas que disponían de una página web corporativa operativa, siendo estas el universo de esta segunda fase.

El formulario se ha distribuido mediante herramientas digitales durante la segunda mitad de 2017 obteniendo un total de 136 respuestas. La muestra obtenida representa el 13,27\% del universo. La recogida, normalización y actualización de estos datos se ha finalizado en 2018, a través del análisis de frecuencias de los resultados obtenidos. 


\section{Resultados}

\subsection{Análisis de contenidos}

La primera parte del análisis responde al objetivo de obtener una radiografía de cuantas de las cooperativas inscritas en el Registro General de Cooperativas de Catalunya disponen de página web, cómo la identifican y si está adaptada a dispositivos móviles.

El primero de los aspectos abordados ha sido el de la presencia en internet a través de una página web corporativa. Teniendo en cuenta las 3.860 cooperativas analizadas encontramos que 1.015 de ellas tienen una página web corporativa publicada en Internet. Esta cifra representa el $27 \%$ del total de cooperativas inscritas en el registro.

Teniendo en cuenta que el $89 \%$ de la muestra de cooperativas catalanas tiene menos de diez socios, el dato de referencia sería el equivalente a las microempresas que, según apunta el informe FAEDPYME (2018), disponen de presencia web el 78,6\% de ellas.

Si analizamos la presencia web por sectores vemos que las cooperativas del sector servicios son las que más presencia web tienen con un $32 \%$, mientras que las cooperativas de la construcción son las que menos presencia web tienen (10\%).

\section{Figura 1. Cooperativas catalanas con página web por sector}

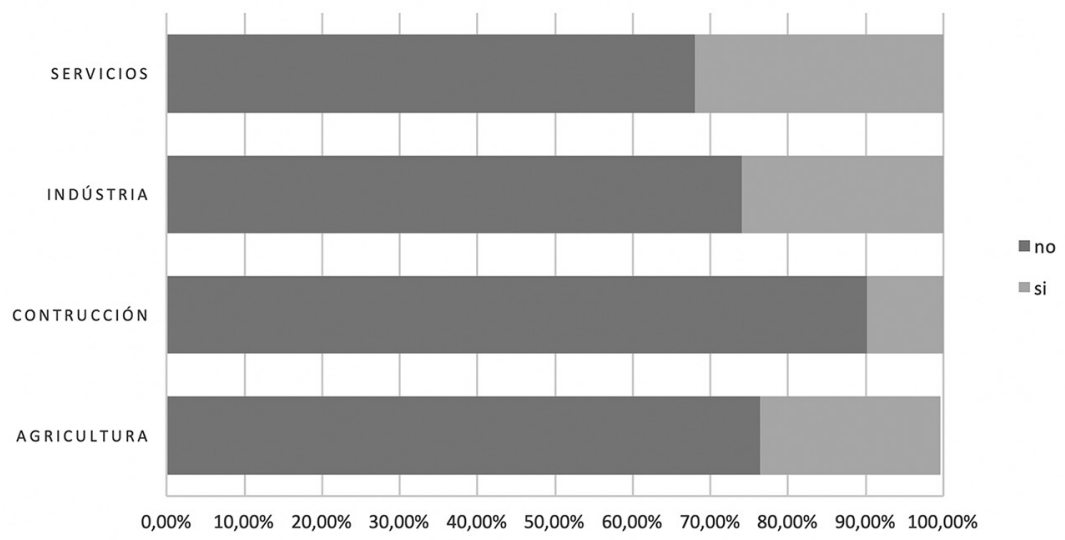


En cuanto a la distribución geográfica de las cooperativas, la provincia de Girona es la que concentra un mayor porcentaje de cooperativas con página web (30,5\%), le siguen las provincias de Barcelona $(27,8 \%)$, Tarragona $(22,3 \%$ y Lleida $(21,9 \%)$. En la siguiente figura podemos observar la distribución de cooperativas con página web por provincia diferenciando entre el sector al que pertenecen.

Tabla 2. Cooperativas catalanas con página web por sector y provincia

\begin{tabular}{|l|c|c|c|}
\hline Província y sector & sin web & con web & Total \\
\hline BARCELONA & $\mathbf{7 2 , 2 0} \%$ & $\mathbf{2 7 , 8 0} \%$ & $\mathbf{1 0 0 , 0 0 \%}$ \\
\hline AGRICULTURA & $72,62 \%$ & $27,38 \%$ & $100,00 \%$ \\
\hline CONSTRUCCIÓN & $91,00 \%$ & $9,00 \%$ & $100,00 \%$ \\
\hline INDÚSTRIA & $74,95 \%$ & $25,05 \%$ & $100,00 \%$ \\
\hline SERVICIOS & $67,29 \%$ & $32,71 \%$ & $100,00 \%$ \\
\hline GIRONA & $\mathbf{6 9 , 4 7 \%}$ & $\mathbf{3 0 , 5 3} \%$ & $100,00 \%$ \\
\hline AGRICULTURA & $76,92 \%$ & $23,08 \%$ & $100,00 \%$ \\
\hline CONSTRUCCIÓN & $83,33 \%$ & $16,67 \%$ & $100,00 \%$ \\
\hline INDÚSTRIA & $57,14 \%$ & $42,86 \%$ & $100,00 \%$ \\
\hline SERVICIOS & $68,24 \%$ & $31,76 \%$ & $100,00 \%$ \\
\hline LLEIDA & $77,88 \%$ & $\mathbf{2 1 , 8 8 \%}$ & $100,00 \%$ \\
\hline AGRICULTURA & $80,69 \%$ & $18,62 \%$ & $100,00 \%$ \\
\hline CONSTRUCCIÓN & $87,76 \%$ & $12,24 \%$ & $100,00 \%$ \\
\hline INDÚSTRIA & $75,00 \%$ & $25,00 \%$ & $100,00 \%$ \\
\hline SERVICIOS & $68,61 \%$ & $31,39 \%$ & $100,00 \%$ \\
\hline TARRAGONA & $77,67 \%$ & $\mathbf{2 2 , 3 3 \%}$ & $100,00 \%$ \\
\hline AGRICULTURA & $65,52 \%$ & $34,48 \%$ & $100,00 \%$ \\
\hline CONSTRUCCIÓN & $90,98 \%$ & $9,02 \%$ & $100,00 \%$ \\
\hline INDÚSTRIA & $73,33 \%$ & $26,67 \%$ & $100,00 \%$ \\
\hline SERVICIOS & $73,06 \%$ & $26,94 \%$ & $100,00 \%$ \\
\hline Total general & $\mathbf{7 3} \%$ & $\mathbf{2 7} \%$ & $\mathbf{1 0 0 \%}$ \\
\hline
\end{tabular}

FUENTE: Elaboración propia. 
El siguiente aspecto analizado ha sido la adaptabilidad del sitio web a dispositivos móviles. Tras analizar las 1.015 páginas web de las cooperativas, vemos como el $52 \%$ de estas sí se encuentran adaptadas a dispositivos móviles según la herramienta de Google.

Figura 2. Páginas web adaptadas a dispositivos móviles

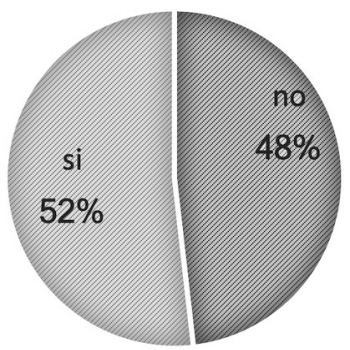

FUENTE: Elaboración propia.

Finalmente, se analiza la identidad en la red de las distintas cooperativas. Encontramos que el dominio .coop solo lo están usando un $6 \%$ de las cooperativas catalanas con página web. La mayoría de las cooperativas apuestan por el uso de dominios .com (46\%), .cat (20\%) y .es (14\%).

\section{Figura 3. Extensiones de dominio usadas en las páginas web de las cooperativas catalanas}

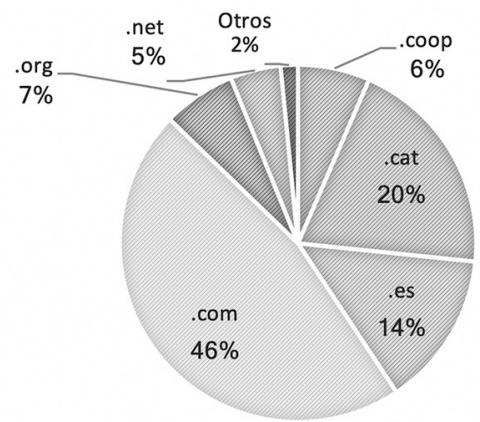

FUENTE: Elaboración propia.

Tal y como hemos podido observar, tanto la penetración de las páginas web en las cooperativas como su adaptación a las políticas mobile first tienen un amplio margen de mejora. 


\subsection{Encuestas}

En segundo lugar, y para complementar la información recogida a través del análisis de contenidos, se analiza el resultado de las encuestas realizadas a las cooperativas con página web. En este sentido, a continuación, se abordan las cuestiones relativas a la finalidad, los elementos clave y las principales barreras para disponer de una página web.

Nueve de cada diez cooperativas afirman utilizar su página web por tres motivos principales: a) mostrar lo que hacen, los productos y los servicios; b) por el simple hecho de tener presencia en la red; c) para que los encuentren.

\section{Figura 4. Principales motivos para tener página web}

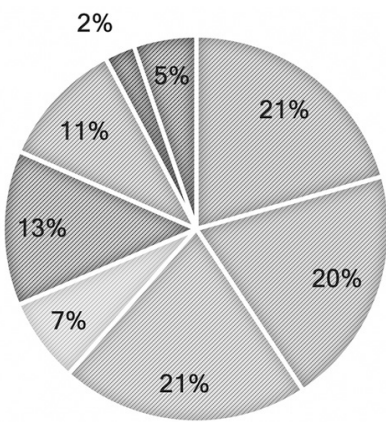

FUENTE: Elaboración propia.

Además, tres de cada cuatro cooperativas con página web afirman que este canal les permite estar más cerca tanto de los clientes como de los proveedores. En una escala de 0 a 5 , siendo 5 muy de acuerdo y 0 muy en desacuerdo, las cooperativas valoran por encima del tres que el hecho de tener página web les facilita la promoción y la venta de productos y servicios, la proximidad y mejora de la comunicación B2B y B2C, y la mejora estratégica. 


\section{Figura 5. Aspectos más importantes de tener presencia web}

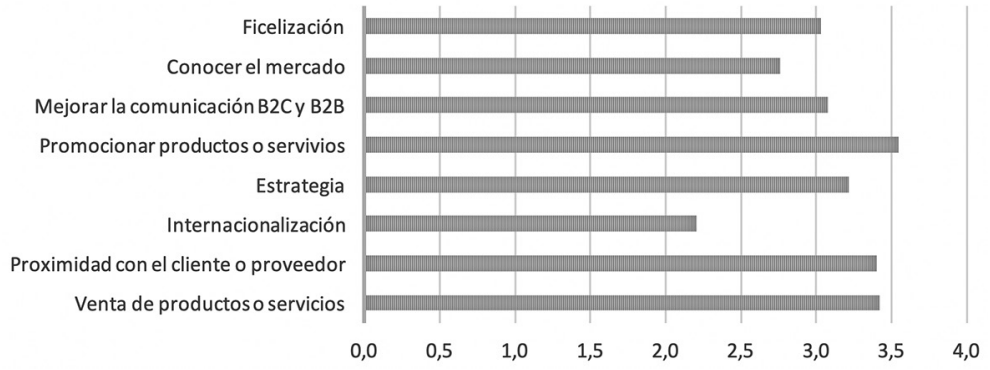

FUENTE: Elaboración propia.

Una de las percepciones que se repiten entre las cooperativas encuestadas es que existe un amplio margen de mejora. Además, al preguntar sobre si el hecho de ser una cooperativa implica alguna diferencia en el uso y la presencia en la red, la respuesta en el $90 \%$ de los casos es que no.

\section{Tabla 3. Percepción de la presencia digital de las cooperativas}

\begin{tabular}{|c|c|}
\hline Número de respuestas & Respuesta \\
\hline 122 & No \\
\hline 12 & $\mathrm{Si}$ \\
\hline 2 & En blanco \\
\hline
\end{tabular}

FUENTE: Elaboración propia.

Entonces, si hay una clara diferencia entre la penetración de la presencia web en las pequeñas y medianas empresas mucho más elevada que la que hay en las cooperativas, ¿cómo se puede ayudar a las cooperativas a estrechar esta brecha? Preguntadas por esta cuestión, las cooperativas apuntan al déficit de recursos como principal motivo. La formación es a su vez un escollo importante para más de la mitad de los encuestadas mientras que el soporte institucional también representa un aspecto clave a mejorar. 


\section{Tabla 4. Percepción de la presencia digital de las cooperativas}

\begin{tabular}{|c|c|}
\hline Número de respuestas & Respuesta \\
\hline 102 & Disponer de más recursos \\
\hline 73 & Tener más formación \\
\hline 51 & Soporte institucional \\
\hline 38 & Tener más información y casos de éxito \\
\hline 9 & Disponer de más tiempo \\
\hline
\end{tabular}

FUENTE: Elaboración propia.

Sin duda, los recursos son un tema nuclear en esta ecuación, siendo especialmente complejo si tenemos en cuenta las dimensiones de la mayoría de las cooperativas catalanas: hablamos de una muestra donde la mayoría de las cooperativas están formadas por tres socios.

\section{Conclusiones y discusión}

Este trabajo ofrece una primera aproximación a la presencia web de las cooperativas en Catalunya. Su elevada muestra nos da una fotografía bastante nítida del estado actual del sector. De todas formas, no se pueden pasar por alto algunas de las limitaciones de este trabajo.

En primer lugar, la composición de la muestra usada. El Registro de Cooperativas de Catalunya se actualiza a partir de la información que recibe y, podría ser, que algunas de las cooperativas presentes en la lista ya no tuvieran ninguna actividad en el momento de realizar el análisis de contenido, aunque no hubiesen notificado la baja en el Registro de Cooperativas de Catalunya.

Y, en segundo lugar, la permanente variación de la información empírica recogida. Este trabajo sigue estando abierto y en permanente revisión. Los datos aportados en 2018 son los que han servido de referente para poder obtener una visión más homogénea del conjunto.

En cualquier caso, y atendiendo a los resultados obtenidos, podemos observar unos niveles muy bajos de cooperativas con página web operativa siendo las cooperativas del sector de la construcción las que menos presencia en la red tienen. Estos datos confirman la baja madurez de la presencia web de las cooperativas observada por Cristóbal et. Al. (2017) en las cooperativas oleicas. 
En relación con el uso del dominio .coop por parte de las cooperativas, confirman la tendencia descrita por Juliá et al. (2004) en relación con el bajo uso del dominio .coop. Una posible línea de investigación futura que no se ha tratado en este trabajo, podría ser conocer el motivo del uso mayoritario de dominios con identificación comercial (.com) o con identificación geográfica (.es y .cat). Quizás esto se deba a una cuestión estratégica en la que se busque un posicionamiento concreto más allá del hecho de ser una empresa constituida como cooperativa.

Otro aspecto a tener en cuenta para explicar los resultados es el tamaño de las cooperativas. Quizá el hecho de que la mayoría de las cooperativas tengan tres socios podría ser un argumento para justificar la falta de recursos para las cuestiones relacionadas con la presencia digital. Es cierto que cuando hablamos de microempresas la presencia web cae más de diez puntos en relación con otras PYME (FAEDPYME, 2018), de todas formas, la diferencia sigue siendo considerable.

Una opción para justificar esta diferencia podría ser argumentar que no son colectivos comparables por la tipología de empresa y por sus finalidades. Según los mismos cooperativistas encuestados estos argumentos tampoco serían válidos puesto que nueve de cada diez afirman que no hay diferencia en el uso e intensidad de la presencia digital por el hecho de ser una cooperativa.

Lo que resulta claro es que hay un amplio margen de mejora. Este podría abordarse desde distintas perspectivas. En primer lugar, y atendiendo a la demanda de los mismos cooperativistas, a través de la formación y la información, por ejemplo, de casos de éxito. ¿Por dónde empezar?

Fortalecer la imagen digital, a través del uso de dominios .coop, ayudaría a posicionar a las cooperativas en su ámbito de actuación, impulsando a la vez al sector en general. Además, una estrategia que podría resultar de utilidad es la apuesta por las políticas mobile first. No solo para acompañar a aquellas cooperativas que ya disponen de página web a adaptarlas sino como estrategia para las que todavía no han dado el paso. 


\section{Bibliografía}

BERELSON, B. (1952): Content Analysis in Communication Research, Michigan, Editora Free Press.

CASTELLS, M. (2001): La galáxia internet, Oxford. Editora Oxford University Press.

CRISÓBAL FRANCI, E., MONTEGUT SALLA, Y. \& DARIES RAMÓN, N. (2017): "Cooperativismo 2.0: Presencia en Internet y desarrollo del comercio electrónico en las cooperativas oleícolas de Cataluña", REVESCO Revista de Estudios Cooperativos, 124, 1-27. https://doi.org/10.5209/ REVE.54926.

DECROP, A. (1999): "Triangulation in Qualitative Research Tourism", Journal of Social Sciences, 20(1), 157-161.

DITRENDIA (2017): Informe ditrendia 2017: Mobile en España y en el Mundo. Disponible online en: https://www.amic.media/media/files/file_352_1289.pdf

FAEDPYME (2018): Análisis estratégico para el desarrollo de la PYME en España. Disponible online en: https://www.cepyme.es/wp-content/uploads/2018/11/informe-FAEDPYME-Espana-2018.pdf.

FERNANDEZ UCLÉS, D., BERNAL JURADO, E., MOZAS MORAL, A., MEDINA VIRUEL, M.J. \& MORAL PAJARES, E. (2015): "El sector cooperativo oleícola y el uso de las TIC: un estudio comparativo respecto a otras formas jurídicas", REVESCO, Revista de Estudios Cooperativos, 120, 53-75. https://doi.org/10.5209/rev_REVE.2016.v120.49700.

HOWE, N. \& STRUASS, W. (2000): Millennials rising: The next great generation, New York, Editora NY Vintage.

I-LabSo SCCL. (2018): V Baròmetre cooperatiu amb valor social. Disponible online en https://xarxanet.org/biblioteca/v-barometre-cooperatiu-amb-valor-social.

ITU (2018): Achieving universal and affordable Internet in the least developed countries. Disponible online en: https://www.itu.int/en/ITU-D/LDCs/Pages/Publications/LDCs/D-LDC-ICTLDC-2018PDF-E.pdf.

JULIÁ IGUAL, J.F., GARCÍA MARTÍNEZ, G. \& MELIÁ MARTÍ, E. (2006): "Internet, cooperativismo agrario y desarrollo rural. El caso de las cooperativas de la Comunidad Valenciana", CIRIEC-España, Revista de Economía Pública, Social y Cooperativa, 55, 221-251. 
JULIÁ IGUAL, J.F., GARCÍA MARTÍNEZ, G. \& POLO GARRIDO, F. (2004): "La información divulgada a través de internet por las cooperativas Gabriel García Martínez y La información divulgada a través de internet por las cooperativas", CIRIEC-España, Revista de Economía Pública, Social y Cooperativa, 49, 167-192.

LAI, K.-W., \& HONG, K.-S. (2015): "Technology use and learning characteristics of students in higher education: Do generational differences exist?", British Journal of Educational Technology, 46(4), 725-738, http://doi.org/10.1111/bjet.12161.

LANIER, J. (2011): Contra el rebaño digital, Madrid: Editora Random house mondadori.

LÓPEZ, N.F. (2002): "El análisis de contenido como método de investigación", XXI Revista de Educación, 4, 167-179.

MOZAS MORAL, A. \& BERNAL JURADO, E. (2004): "Integración cooperativa y TIC's: presente y futuro", CIRIEC-España, Revista de Economía Pública, Social y Cooperativa, 49, 143-166.

ONTSI (2020): Dossier de indicadores sobre uso TIC en PYMES en España y la UE, Disponible online: https://www.ontsi.red.es/sites/ontsi/files/2020-05/DossierPYMESMayo2020.pdf.

PALACIOS, M. \& DÍAZ NOCI, J. (2009): Ciberperiodismo: métodos de investigación. Una aproximación multidisciplinar en perspectiva comparada, Editora Servicio editorial de la Universidad del Pais Basco.

PALFREY, J. \& GASSER, U. (2008): Born digital, New York, Editora Basic Books.

PEW RESEARCH (2018): Mobile Fact Sheet. Disponible online en: https://www.pewresearch.org/internet/fact-sheet/mobile/

PRENSKY, M. (2001): "Digital Natives, Digital Immigrants", On the Horizon, 9(5).

TAPSCOTT, D. (1998): Growing up digital. The rise of the Net Generation, New York, Editora McGraw Hill.

VERISIGN (2019): "The domain name industry brief - Q1 2019", The Domain Name Industry Brief, 16(2), $2-5$.

WHITE, D.S. \& LE CORNU, A. (2011): "Visitors and Residents: A new typology for online engagement", First Monday, 16(9). 


\section{Anexo 1. Variables del análisis de contenido}

Para la primera exploración se han usado los siguientes datos secundarios extraídos del "Registre General de Cooperatives de Catalunya":

- Nombre de la sociedad

- Tipo de sociedad

- Fecha de inscripción

- CCAE principal

- Descripción CCAE

- Sector

- Codigo postal

- Municipio

- Comarca

- Provincia

- Clase de cooperativa

Estos datos se han complementado con la obtención de los siguientes datos primarios a través del análisis de contenidos:

- Dirección de correo electrónico

- ¿Tiene web corporativa? (si/no)

- URL de la Web

0 Identificación de la web (dominio)

- Adaptación de la web a dispositivos móviles 


\section{Anexo 2. Esquema de las entrevistas}

Bloque de control: El objetivo de este bloque es validar los datos obtenidos en la fase del análisis de contenido.

- Nombre de la cooperativa

- ¿Vuestra cooperativa tiene web?

- En caso AFIRMATIVO, ¿nos puede indicar la URL?

- En caso AFIRMATIVO, ¿puede indicarnos los motivos?

- En caso NEGATIVO, ¿puede indicarnos los motivos?

Bloque valorativo: El objetivo de este bloque es recoger la valoración de cada cooperativa sobre el uso de su página web

- Valora de 1 a 5 la importancia de la web de vuestra cooperativa en relación con los siguientes aspectos:

- [Venta de productos o servicios]

- [Proximidad con el cliente/ proveedor]

○ [Internacionalización]

- [Estrategia]

- [Promoción de productos y/o servicios]

- [Mejora de la comunicación B2B y B2C]

- [Conocimiento del mercado]

- [Fidelización]

- Valora de 1 a 5 el aprovechamiento de la web de vuestra cooperativa en relación con los siguientes aspectos:

○ [Valoración]

- ¿Cuál de los siguientes factores pueden ayudar a mejorar el aprovechamiento de la red

- por parte de vuestra cooperativa?

- [Disponer de más recursos]

- [Disponer de más tiempo]

- [Más soporte institucional]

- [Tener más formación]

- [Tener más información y casos de éxito]

Bloque de percepción: El objetivo de este bloque es ver si perciben alguna diferencia en relación al aprovechamiento de una web corporativa por el hecho de ser una cooperativa:

- ¿Crees que el hecho de ser una cooperativa condiciona el hecho de tener presencia en la red?

○ ¿Por qué? 